\title{
The principle of general tovariance
}

\author{
C. Heunen* $\quad$ N.P. Landsman ${ }^{\dagger} \quad$ B. Spitters ${ }^{\ddagger}$
}

March 7, 2008

\begin{abstract}
We tentatively propose two guiding principles for the construction of theories of physics, which should be satisfied by a possible future theory of quantum gravity. These principles are inspired by those that led Einstein to his theory of general relativity, viz. his principle of general covariance and his equivalence principle, as well as by the two mysterious dogmas of Bohr's interpretation of quantum mechanics, i.e. his doctrine of classical concepts and his principle of complementarity. An appropriate mathematical language for combining these ideas is topos theory, a framework earlier proposed for physics by Isham and collaborators.

Our principle of general tovariance states that any mathematical structure appearing in the laws of physics must be definable in an arbitrary topos (with natural numbers object) and must be preserved under so-called geometric morphisms. This principle identifies geometric logic as the mathematical language of physics and restricts the constructions and theorems to those valid in intuitionism: neither Aristotle's principle of the excluded third nor Zermelo's Axiom of Choice may be invoked. Subsequently, our equivalence principle states that any algebra of observables (initially defined in the topos Sets) is empirically equivalent to a commutative one in some other topos.
\end{abstract}

\section{Motto}

'At a certain point in its history, the fundamental problems of physics have to do with the way in which fundamental concepts are defined. In those circumstances, the pursuit of physics in accord with those concepts evidently has not resolved the underlying problems. These are the times at which philosophical analysis has become an unavoidable task of physics itself.'

(R. DiSalle [9])

Dedicated to Klaus Fredenhagen, at his 60th birthday

\footnotetext{
${ }^{*}$ Radboud Universiteit Nijmegen, Institute for Computing and Information Sciences, Toernooiveld 1, 6525 ED NIJMEGEN, THE NETHERLANDS

${ }^{\dagger}$ Radboud Universiteit Nijmegen, Institute for Mathematics, Astrophysics, and Particle Physics, Toernooiveld 1, 6525 ED NIJMEGEN, THE NETHERLANDS

${ }^{\ddagger}$ Eindhoven University of Technology, Department of Mathematics and Computer Science, P.O. Box 513, 5600 MB EINDHOVEN, THE NETHERLANDS
} 


\section{Old principles}

After decades of stagnation [28, 30], the possibility suggests itself that the present difficulties in constructing a satisfactory quantum theory of gravity reflect insufficient understanding of the foundations of quantum mechanics and its relationship to general relativity. In fact, our knowledge of the foundations of both theories is questionable and one can hardly expect a successful merge before these foundations have been clarified. Thus instead of taking quantum theory at face value and following the "shut up and calculate" philosophy common in string theory and (less so) in loop quantum gravity, we believe that the road to progress lies in entering a process of clarifying the foundations of quantum theory and general relativity, preferably in interaction with each other. This process might eventually suggest modifications to both theories even at the level at which they are currently defined (i.e. quantum theory without gravity and classical general relativity).

Even without the desire to incorporate general relativity, in quantum theory the emergence of classicality is a major concern [21]. In our opinion, this issue will have to be understood in conjunction with the question of (in)determinism in quantum mechanics; these are two sides of the same coin. Without this joint perspective, the relationship between the logical and the probabilistic structure of quantum mechanics is obscure. ${ }^{1}$ The present paper, which was partly inspired by the papers of Isham and collaborators $[15,10]$ and partly by thoughts on general relativity (as suggested above), is meant as a first step towards the clarification of the relationship in question. It seems inconceivable that a successful theory of quantum gravity can be found before this has been done.

To start with general relativity, it is well known that Einstein, despite being single-handedly responsible for one of the high points in human thought in formulating the theory of general relativity, was actually somewhat confused about the foundations of his own theory (see [16] for most of the original papers and cf. [25] for a survey of both the primary and the secondary literature). On his road to general relativity, Einstein was guided by three principles: the principle of general covariance, the equivalence principle and Mach's principle. All three are controversial, but the latter seems particularly ill-founded and Einstein eventually abandoned it himself. It will play no role in our considerations.

The principle of general covariance was originally meant by Einstein as an expression of the idea of the general relativity of motion, but this idea is flawed. In fact, general relativity singles out geodesics as preferred carriers of motion, much like the inertial frames in Newtonian mechanics and special relativity. Moreover, general covariance has been claimed to be vacuous. This, however, is an exaggeration: general covariance delineates the mathematical framework of Einstein's theory as differential geometry. Ironically, this framework applies to classical mechanics and special relativity as well; the essence of general relativity lies in the dynamical role of the space-time metric.

An appropriate version of the equivalence principle states that free fall in a gravitational field is locally indistinguishable from rest or uniform motion in Minkowski space-time without gravitational forces, and hence locally free fall defines an inertial frame. Although also this principle has been derided, we follow Einstein himself as well as the rational reconstruction of his thought by DiSalle [9] in attaching great importance to it. Namely, the equivalence principle makes the Newtonian separation between inertial motion and acceleration ill-defined in case that the latter is caused by gravity, and furthermore it leads to the seemingly paradoxical feature that in the presence of gravity different locally inertial frames are not inertial with respect to each other (as they would be without gravity). In combination with general covariance, i.e. the geometric framework of the theory, the equivalence principle then enables one to solve both problems at one stroke by identifying inertial motion in a gravitational field with geodesic motion in a curved space-time. All that remained for Einstein was to find the relationship between curvature and matter distribution, a (still Herculean) task he accomplished by discovering the equations now named after him.

Einstein's great antagonist was Bohr. His understanding of quantum mechanics was expressed by two principles: the doctrine of classical concepts and the principle of complementarity (see [18] for the original papers and [27] for penetrating analysis). Significantly, whereas Einstein had his principles before his theory, Bohr formulated them after the completion of quantum mechanics. Nonetheless, his

\footnotetext{
${ }^{1}$ The quantum logic of Birkhoff and von Neumann $[5,8]$ has actually clarified neither the logical foundation of quantum theory nor its probabilistic structure. Indeed, its lack of distributivity and of a satisfactory implication, as well as its inherent propositional (as opposed to predicate) form has defied a reasonable interpretation and application of quantum logic. These difficulties reflect the lack of integration of quantum logic with modern logic, especially with categorical logic and topos theory $[17,22]$. Also, the goal of deriving quantum theory from quantum-logical axioms has not been achieved, despite impressive attempts [26].
} 
principles are even more obscure than Einstein's, and in fact it seems that only some people who have known Bohr personally still endorse them. This is a pity, because in our opinion the door to quantum gravity is opened by reformulating Bohr's principles of quantum mechanics in a way inspired by Einstein's principles of gravity. $^{2}$ To do so, we turn to topos theory. ${ }^{3}$

\section{What is a topos?}

According to McLarty [23], 'Elementary toposes arose when Lawvere's interests in the foundations of physics and Tierney's in the foundations of topology led both to study Grothendieck's foundations for algebraic geometry. ${ }^{4}$ Topos theory has quite appropriately been compared with an elephant as regards size, complexity, and the possibility of adopting seemingly different points of view [17]. Indeed, the first sentence of the well-known book by Mac Lane and Moerdijk on topos theory [22] is this:

'A startling aspect of topos theory is that it unifies two seemingly wholly distinct mathematical subjects: on the one hand, topology and algebraic geometry and on the other hand, logic and set theory.'

This is elaborated by Vickers [29], as follows:

'We have, on the one hand, the logic and the set theory, and, on the other, the topology. In a nutshell, the topos connection between them is that the topos acts like a "Lindenbaum algebra" (of formulae modulo equivalence) for a logical theory whose models are the points of a space.'

Indeed, topoi provide semantics for intuitionistic predicate logic, and as such generalize frames (or, equivalently, Heyting algebras) as the appropriate semantics for intuitionistic propositional logic [29]. ${ }^{5}$ Here one looks at frames as the Lindenbaum algebras of intuitionistic propositional logic. ${ }^{6}$ Thus a topos is something like the "Lindenbaum algebra" (technically, the classifying topos) of some intuitionistic predicate logic [29].

Most presentations of the subject, however, start from the idea that topoi generalize the category Sets of sets and functions. This approach defines a topos as a category with:

1. Terminal object; 2. Exponentials; 3. Pullbacks; 4. Subobject classifier.

These generalize, respectively, the following constructions in Sets:

1. An arbitrary singleton 1 (in that from any set $X$ there is a unique arrow $X \rightarrow 1$ );

2. Sets $Y^{X}$ consisting of all functions $X \stackrel{f}{\rightarrow} Y$, for arbitrary sets $X$ and $Y$;

3. Fibered products $B \times{ }_{A} C=\{(b, c) \in B \times C \mid f(b)=g(c)\}$ of $B \stackrel{f}{\rightarrow} A$ and $C \stackrel{g}{\rightarrow} A$, along with the obvious projection maps $B \leftarrow B \times{ }_{A} C \rightarrow C$;

4. The set $\Omega=\{0,1\}$ in its role as the codomain of characteristic functions of subsets: given an inclusion $A \subset B$ of sets, one can reconstruct $A$ from its characteristic function $B \stackrel{\chi_{A}}{\rightarrow} \Omega$ and, vice versa, $\chi_{A}$ is of course defined by $A$.

In a general topos, $\Omega$ comes with an arrow $1 \stackrel{\top}{\rightarrow} \Omega$ called truth, such that for every monomorphism $A \stackrel{f}{\rightarrow} B$ (i.e. injection in the categorical sense, often denoted by $A \longmapsto B$ ) there is a unique arrow $B \stackrel{\chi_{A}}{\rightarrow} \Omega$

\footnotetext{
${ }^{2}$ See also [20] for a different attempt at a peaceful resolution to the Bohr-Einstein debate.

${ }^{3}$ To get familiar with topos theory, it is advisable to study $[12,22,17]$ in the given order.

${ }^{4}$ See also [19] for a general history of category theory. In the original work of Grothendieck [1], topoi generalize topological spaces, in that open sets are generalized by "set-valued" opens, or sheaves. Lawvere's ideas on the connection with physics are elaborated in [3].

${ }^{5} \mathrm{~A}$ frame is a sup-complete distributive lattice such that $x \wedge \vee_{\lambda} y_{\lambda}=\vee_{\lambda} x \wedge y_{\lambda}$. For example, if $X$ is a topological space, then the topology (i.e. the set of open sets) $\mathcal{O}(X)$ of $X$ is a frame with $U \leq V$ if $U \subseteq V$. Frames are sometimes called locales, but the category of locales is the opposite category to the category of frames. This seemingly curious convention guarantees that, in the above example, a continuous map $f: X \rightarrow Y$ induces a map $f_{*}: \mathcal{O}(X) \rightarrow \mathcal{O}(Y)$ between the associated locales, defined as $f^{*} \equiv f^{-1}: \mathcal{O}(Y) \rightarrow \mathcal{O}(X)$ read in the opposite direction. The mind boggles!

${ }^{6}$ These are given by the set of formulae modulo provable equivalence, ordered by entailment.
} 
for which $B \stackrel{f}{\leftarrow} A \rightarrow 1$ is a pullback of $B \stackrel{\chi_{A}}{\rightarrow} \Omega$ and $1 \stackrel{\top}{\rightarrow} \Omega .{ }^{7}$ Amazingly, $\Omega=\{0,1\}$ in its role as the subobject classifier in the topos Sets carries the entire logical structure of classical mathematics. In general, in an arbitrary topos $\Omega$ is a so-called Heyting algebra, which provides an algebraic description of some intuitionistic propositional logic intrinsic to the topos at hand. In such a logic, neither Aristotle's principle of the excluded third, ${ }^{8}$ nor Zermelo's Axiom of Choice necessarily holds. ${ }^{9}$

\section{States, propositions and truth}

The logical perspective on topos theory, culminating in the decisive role of the subobject classifier, explains its potential relevance for physics. For, as emphasized by Isham and collaborators [15, 10], the topos framework leads to a new way of thinking about truth in quantum mechanics. Traditionally, the mathematical arena of both classical and quantum physics has - in blissful ignorance - simply been the topos Sets. Both are based on a pairing $\langle\omega, P\rangle \mapsto[0,1]$, where $\omega$ is a state and $P$ is an elementary proposition (or "yes-no question"). The number $\langle\omega, P\rangle$ equals the probability that the proposition $P$ is true in the state $\omega$. Elementary propositions are of the form $P=a \in U$, where $a$ is an observable and $U \subset \mathbb{R}$. It will be sufficient to deal with the case $U=(r, s)$; more generally, one may assume that $U$ is regular open (i.e. $U$ coincides with the interior of its closure) ${ }^{10}$ In classical physics, this scheme works fine. An observable $a$ is a real-valued function $M \stackrel{a}{\rightarrow} \mathbb{R}$ on some phase space $M$, and a yes-no question $a \in U$ may be identified with the subset $[a \in U]=a^{-1}(U) \subset M$. (More precisely, $a \in U$ should be identified with the equivalence class of all propositions $b \in V$ with the same inverse image $b^{-1}(V)=a^{-1}(U)$.) This subset is open if $a$ is continuous, and defines a monomorphism $a^{-1}(U) \stackrel{P}{\longmapsto} M$, characterized by its classifying arrow $M \stackrel{\chi_{a}-1(U)}{\longrightarrow} \Omega$. A pure state $\omega$ is just a point of $M$, hence a monomorphism $1 \stackrel{\omega}{\rightarrow} M$. Composition of $\omega$ and $\chi_{a^{-1}(U)}$ yields an arrow

$$
1 \stackrel{\langle\omega, P\rangle}{\longrightarrow} \Omega=1 \stackrel{\omega}{\longrightarrow} M \stackrel{\chi_{a-1}(U)}{\longrightarrow} \Omega
$$

which is precisely the truth value of the proposition $P=a \in U$ in the state $\omega$, i.e. $\langle\omega, P\rangle$ is true or equal to 1 if $\omega \in a^{-1}(U)$ (or $a(\omega) \in U$ ) and false or equal to 0 otherwise.

Thus the subobject classifier

$$
\Omega=\{0,1\}=\{\perp, \top\}=\{\text { false, true }\}=\{\text { no, yes }\}
$$

in the topos Sets is the carrier of truth values of yes-no questions, the truth (i.e. answer "yes") or falsehood (i.e. answer "no") of such questions being assigned by some pure state. If the state is mixed, the truth value of a yes-no question is a point in $[0,1]$, interpreted as the probability that the answer to the question is "yes." This is unproblematic in classical physics, as the decomposition of a mixed state $\omega$ into pure components leads to an associated decomposition of the pairing $\langle\omega, P\rangle$ into terms that are either equal to 0 or to 1 .

From a localic point of view, one rather works with the topology $\mathcal{O}(M)$ instead of with $M$ itself. Still working in Sets, our yes-no question $P=a \in U$ is just an arrow $1 \stackrel{a^{-1}(U)}{\longrightarrow} \mathcal{O}(M) \equiv 1 \stackrel{P}{\rightarrow} \mathcal{O}(M)$. A pure state $\omega$ is now represented by the subobject $S_{\omega} \subset \mathcal{O}(M)$ given by

$$
S_{\omega}=\{V \in \mathcal{O}(M) \mid \omega \in V\} .
$$

The pairing map becomes

$$
1 \stackrel{\langle P, \omega\rangle}{\longrightarrow} \Omega=1 \stackrel{P}{\longrightarrow} \mathcal{O}(M) \stackrel{\chi_{S_{\omega}}}{\longrightarrow} \Omega,
$$

\footnotetext{
${ }^{7}$ For example, in the topos $\operatorname{Sh}(X)$ of sheaves over a topological space $X$ the subobject classifier is the functor $U \mapsto \mathcal{O}(U)$ acting on arrows (i.e. inclusions) $U \leq V$ as $\mathcal{O}(V) \rightarrow \mathcal{O}(U), W \mapsto W \cap U$.

${ }^{8}$ In the example $\operatorname{Sh}(X)$ the logical operations on the internal Heyting algebra $\Omega: U \mapsto \mathcal{O}(U)$ are computed pointwise: truth $1 \stackrel{\top}{\rightarrow} \Omega$ is $\top=U$, false $1 \stackrel{\perp}{\rightarrow} \Omega$ is $\perp=\emptyset$, negation $\neg: \Omega \rightarrow \Omega$ is $\neg V=\operatorname{int}(U \backslash V)$, conjunction $\wedge: \Omega \times \Omega \rightarrow \Omega$ is $V \wedge W=V \cap W$, disjunction $\vee: \Omega \times \Omega \rightarrow \Omega$ is $V \vee W=V \cup W$, and finally (material) implication $\Rightarrow: \Omega \times \Omega \rightarrow \Omega$ reads $V \Rightarrow W=\operatorname{int}((U \backslash V) \cup W)$. Consequently, $V \vee \neg V=U \backslash \partial V \neq U=\top$.

${ }^{9}$ In the sense that any epimorphism, i.e. categorical surjection, has a right inverse.

${ }^{10}$ If one admits general open sets, the logic of classical mechanics already comes out as intuitionistic! This is because the open subsets of a topological space do not form a Boolean algebra under natural operations.
} 
where $\chi_{S_{\omega}}$ is the classifying map of $S_{\omega} \longmapsto \mathcal{O}(M)$. Of course, one has $\langle P, \omega\rangle=\langle\omega, P\rangle$; cf. (1). It is this localic formulation of the state-proposition pairing that can be generalized to quantum theory, as follows. ${ }^{11}$

Let $\mathrm{C}(\mathfrak{A})$ be the collection of commutative unital $C^{*}$-subalgebras of a unital $C^{*}$-algebra $\mathfrak{A}$, partially ordered by inclusion (seen as a category in the usual way) and let $\mathbf{T}(\mathfrak{A})$ be the topos $\operatorname{Sets}^{\mathrm{C}(\mathfrak{A})}$ of functors $\mathrm{C}(\mathfrak{A}) \rightarrow$ Sets. The map that sends $\mathfrak{C}$ (seen as an object in $\mathrm{C}(\mathfrak{A})$ ) into itself (seen as a set) is functorial in the obvious way and defines an object $A$ in $\mathbf{T}(\mathfrak{A})$. This object is a commutative $C^{*}$-algebra in $\mathbf{T}(\mathfrak{A})$ in a natural way. ${ }^{12}$ We call $A$ the Bohrification of $\mathfrak{A}$. There is a Gelfand theory for commutative $C^{*}$-algebras in topoi [2], ${ }^{13}$ and the Gelfand spectrum $\Sigma$ of $A$ is automatically a (compact completely regular) locale.

This locale, which is explicitly described in [14], plays a central role in the logical analysis of the quantum theory defined by $\mathfrak{A}$. Being a locale, it is automatically a Heyting algebra, carrying the intuitionistic propositional logic intrinsic to the theory. This logic is 'spatial' in the sense that its elementary propositions correspond to arrows $1 \rightarrow \Sigma$; as in the classical case $\Sigma=\mathcal{O}(M)$, we regard such arrows as open subsets of the space that formally underlies the locale $\Sigma$. The relationship between the original observables of the system and such propositions is not obvious, ${ }^{14}$ but it turns out that, as in classical physics, a self-adjoint element $a \in \mathfrak{A}$ and an interval $(r, s) \subset \mathbb{R}$ define an arrow (i.e. 'open set') $1 \stackrel{[a \in(r, s)]}{\longrightarrow} \Sigma$. In addition, one may generalize (3) in associating a subobject of $\Sigma$ to a state $\omega$ on $\mathfrak{A}[7,14]$. At the end of the day, this leads to pairing map defined exactly as in (4), but now taking values in the subobject classifier of the topos $\mathbf{T}(\mathfrak{A})$ at hand.

This suggests that, quite generally, the subobject classifier plays the role of the codomain of the pairing map between (pure) states and yes-no questions. Thus the answers to such questions are neither limited to "yes" or "no", nor to some number in the interval $[0,1]$ playing the role of some probability. Instead, as suggested in [15, 10], truth becomes an - a priori non-probabilistic - multi-valued notion in non-classical theories of physics like quantum mechanics. It is precisely the attraction of topos theory for physics that this theory provides a formal setting for such an idea. ${ }^{15}$

Truth is prior to probability, and the next step would consist in deriving the notion of ordinary probability and the Born rule from multi-valued truth assignments in a suitable topos.

\section{New principles}

Having introduced the notion of a topos and its motivation for physics, we wish to return to the task of finding suitable principles that might underlie quantum gravity. Before doing so, however, we need one more ingredient of topos theory [17, 22, 29].

So far, we have seen that topoi generalize frames (the generalization being from propositional to predicate logic), which in turn generalize topological spaces: such a space $X$ defines the frame $\mathcal{O}(X)$ of all open sets, but not all frames are of this form. Nonetheless, the notion of a map between frames is taken from this example: if $f: X \rightarrow Y$ is continuous, then the induced map $f^{-1}: \mathcal{O}(Y) \rightarrow \mathcal{O}(X)$ is a lattice morphism preserving finite $\wedge$ and all $\vee$. Hence also in general, frame maps are defined in this way. It is clear from the definition of a frame that such maps are quite natural, but they have the disadvantage that they do not preserve the entire logical (i.e. Heyting algebra) structure encoded in a frame: (material) implication and hence negation are not necessarily preserved. Recalling that frames are Lindenbaum algebras of intuitionistic propositional logics, this problem may be circumvented by

\footnotetext{
${ }^{11}$ Our construction was inspired by the work of Döring and Isham [10], from which it differs in several respects. For example, whereas Döring and Isham work with spaces, we work with locales. This is a mathematical reflection of the difference between the philosophical ideas of Heidegger [10] and Bohr [14].

${ }^{12}$ In a paper dedicated to Klaus Fredenhagen, it may be appropriate to point out that an algebraic quantum field theory may not just be seen as a functor, as in [6], but as a single $C^{*}$-algebra in the topos of pre-cosheaves over space-time, by a similar construction as in the main text.

${ }^{13}$ The Gelfand spectrum $\Sigma$ of $A$, seen as a frame or locale, is the Lindenbaum algebra of the logical theory defined by propositions of the form $a \in U$ indexed by $a \in A$ and $U \in \mathcal{O}(\mathbb{C})$, subject to relations suggested by the situation in Sets. One then has an isomorphism $A \cong C(\Sigma)$, where $C(\Sigma)$ stands for the set of locale maps from $\Sigma$ to $\mathcal{O}(\mathbb{C})$ and hence of frame maps in the opposite direction. The Gelfand transform of $a \in A$ is $\hat{a}: \mathcal{O}(\mathbb{C}) \rightarrow \Sigma, U \mapsto[a \in U]$ (i.e. the equivalence class of the proposition $a \in U$, which by definition of $\Sigma$ is an element of it).

${ }^{14}$ The difficulty lies in associating an element of $A$ to one of $\mathfrak{A}$. This is achieved in [14] by a modification of the 'Daseinization' map of Döring and Isham [10].

${ }^{15}$ There is a clear parallel with model theory here, in that through the pairing map each state $\omega$ defines a model of the logical theory underlying the spectral object $\Sigma$ (seen as a frame or Heyting algebra) taking values in $\Omega$. This generalizes the Boolean models of classical logic, which in turn generalize the simplest models of logic taking values in $\{0,1\}=\{\perp, \top\}$.
} 
only allowing such logical theories that in addition are geometric. This curious name (whose origin lies in fact in the geometric side of topos theory) stands for the fragment of intuitionistic propositional logic whose formulae are of the form $\varphi \rightarrow \psi$, where $\varphi$ and $\psi$ are built from atomic propositions using the symbols $T$ (for "truth"), $\wedge$ (for "and"), and $\vee$ (for "or"), where $\vee$ but not $\wedge$ is allowed to carry an infinite index set. This may be motivated by the remark that to verify a proposition $\vee_{\lambda \in \Lambda} p_{\lambda}$ one only needs to find a single $p_{\lambda}$, whereas to verify $\wedge_{\lambda \in \Lambda} p_{\lambda}$ the truth of each $p_{\lambda}$ needs to be established, an impossible task in practice when $\Lambda$ is infinite. One may then say that frame maps preserve "geometric" propositional logical theories.

This idea generalizes to predicate logic, and hence to topos theory. A geometric intuitionistic predicate logic is a theory whose formulae are as above, ${ }^{16}$ now also involving the existential quantifier $\exists$, and whose axioms take the form $\forall x: \varphi(x) \rightarrow \psi(x) .{ }^{17}$ The generalization of a frame map to topos theory is, then, a so-called geometric morphism, whose reason of existence is to preserve "geometric" first order theories. Specifically, a geometric morphism $F \rightarrow E$ is functor $\phi_{*}: F \rightarrow E$ with left adjoint $\phi^{*}: E \rightarrow F$ that preserves finite limits (and also, automatically as a left adjoint, all colimits). Hence the 'inverse image part' $\phi^{*}$ of a geometric morphism preserves any mathematical structure in a topos that is axiomatized "geometrically" in the sense just described; it may be seen as the ultimate generalization of a continuous map between topological spaces. Thus geometric morphisms form the natural maps between topoi.

For the simplest examples of geometric morphisms, consider the presheaf topos Sets $^{\mathrm{C}}$ of functors from a category $\mathrm{C}$ to Sets. Then for each $C \in \mathrm{C}$ one has a geometric morphism $\phi_{C}:$ Sets $\rightarrow$ Sets $^{\mathrm{C}}$ with inverse image $\phi_{C}^{*}=\mathbf{e v}_{C}:$ Sets $^{\complement} \rightarrow$ Sets, i.e. the evaluation map given by $\mathbf{e v}_{C}(F)=F(C)$. In particular, for $\mathrm{C}=\mathrm{C}(\mathfrak{A})$ and $F=A$ one obtains $\mathbf{e v}_{C}(A)=C$. Hence selecting a commutative $C^{*}$-subalgebra $C \subset \mathfrak{A}$ from the Bohrification $A$ of $\mathfrak{A}$ is a geometric operation; physically, this picks a classical context. ${ }^{18}$ In the opposite direction, there is a unique geometric morphism $\psi:$ Sets $^{C} \rightarrow$ Sets with inverse image $\psi^{*}$ : Sets $\rightarrow$ Sets $^{\mathcal{C}}$ given as the constant functor $\psi(X): C \mapsto X$ for all $C \in \mathbf{C}$.

Einstein's principle of general covariance then has a natural analogue in topos theory, which we call the principle of general tovariance: any mathematical structure appearing in the laws of physics must be definable in an arbitrary topos (with natural numbers object) and must be preserved under geometric morphisms. Like the principle of general covariance, the principle of general tovariance has no immediate physical content, but merely serves to identify the mathematical language of physics. Where general covariance meant differential geometry, general tovariance implies geometric logic. ${ }^{19}$ In particular, it restricts the mathematical objects that may occur in physics to those whose core can be axiomatized through geometric logic. It seems to us that this requirement is the correct formalization of the familiar idea that physics at the Planck scale be finite (or at least discrete). Our principle also expresses the idea that physics should be as independent as possible of the mathematical framework (to the extent that different topoi stand for different mathematical universes). ${ }^{20}$

Which mathematical theories are "geometric" in this sense? As far as physically relevant structures are concerned, we mention differential geometry, albeit in algebraic form (i.e. instead of smooth manifolds $M$ one works with the function $\operatorname{ring} C^{\infty}(M)$, etc.) [24], as well as pre-quasi-C $C^{*}$-algebras. These are "almost" $C^{*}$-algebras, except that the norm is actually a semi-norm and that the underlying space may not be complete. ${ }^{21}$ The usual process of turning a pre-quasi- $C^{*}$-algebra into an actual $C^{*}$-algebra by quotienting by the zero-norm subspace and completing turns out to be nongeometric, ${ }^{22}$ but this is of little concern, because what matters is a given, "coordinate invariant" theory, which may be turned into

\footnotetext{
${ }^{16}$ The atomic formulae may now involve relations and equalities and all the usual structures allowed in first-order logic as well.

${ }^{17}$ In both cases deductions $\left\{\varphi_{1}, \ldots, \varphi_{n}\right\} \rightarrow \psi$ are made from lists of finitely many formulae to single formulae. See [29] and [17] for details.

${ }^{18}$ Continuing footnote 12 , the map $\mathcal{O} \mapsto \mathfrak{A}(\mathcal{O})$ defining an algebraic quantum field theory comes out as geometric in exactly the same way.

${ }^{19}$ Thus the tendentious slogan that "physics is information" has to be replaced by the idea that physics is logic.

${ }^{20}$ From this perspective the situation is not just similar to general relativity, but also to algebraic quantum field theory. Indeed, passing from a Hilbert space framework to a $C^{*}$-algebraic one captures the idea of the representation-independence of the underlying theory, a point emphasized by Haag and Kastler [13] through their appeal to Fell's theorem [11]: If $\mathfrak{A}$ is a simple $C^{*}$-algebra and $\pi$ is a representation, the normal states on $\pi(\mathfrak{A})$ are weak* dense in the state space of $\mathfrak{A}$. Hence according to Haag and Kastler all representations of $\mathfrak{A}$ are 'physically equivalent'.

${ }^{21}$ Since suprema and infima may not exist in an arbitrary topos, one has to look at the seminorm in an unusual way; roughly speaking, it is defined by specifying $N(q)=\{a \in A \mid\|a\|<q\}$, where $q$ is rational, instead of $\|\cdot\|$ itself.

${ }^{22}$ Equivalently, applying the inverse image part of a geometric morphism to a $C^{*}$-algebra fails to preserve completeness and the definiteness of the norm. We are indebted to Chris Mulvey for explaining this point.
} 
an (internal) $C^{*}$-algebra in an arbitrary topos by the said, "non-invariant" procedure.

We are now in a position to generalize Bohr's doctrine of classical concepts in a way reminiscent of Einstein's equivalence principle: we postulate that any algebra of observables is empirically indistinguishable from a commutative one. ${ }^{23}$ Much as an observer can locally avoid the gravitational force by moving along a geodesic (which in Einstein's view amounted to a special choice of coordinates), an observer in quantum theory can get rid of the noncommutativity of a $C^{*}$-algebra of observables by moving to a very special topos in which it becomes commutative. ${ }^{24}$ This sounds paradoxical, and indeed it has been suggested that Bohr was simply incompetent [4]. However, we have seen in the previous section that the above equivalence principle can indeed be implemented [14]. The use of topos theory then automatically takes care of complementarity, in the sense that mutually exclusive classical snapshots of reality naturally combine into a single internal commutative $C^{*}$-algebra, which carries all physical information. ${ }^{25}$

Note added: Following talks by the second author at Lisbon, Princeton and Hamburg in the Fall of 2007, an interesting discussion on General Tovariance has emerged in the n-Category Café http://golem.ph.utexas.edu/category, see December 5, 2007. We hope this paper clarifies the main issues raised there.

\section{References}

[1] M. Artin, A. Grothendieck \& J.-L. Verdier, Théorie de topos et cohomologie étale des schémas (SGA4), Lecture Notes in Mathematics 269, 270. Springer, Berlin, 1972.

[2] B. Banaschewski \& C.J. Mulvey, A globalisation of the Gelfand duality theorem. Ann. Pure Appl. Logic 137, 62-103 (2006).

[3] J.L. Bell, A primer of infinitesimal analysis. Cambridge University Press, Cambridge, 1998.

[4] M. Beller, Quantum dialogue. University of Chicago Press, Chicago, 1999.

[5] G. Birkhoff \& J. von Neumann, The logic of quantum mechanics. Ann. Math. 37, 823-843 (1936).

[6] R. Brunetti, K. Fredenhagen \& R. Verch, The generally covariant locality principle - A new paradigm for local quantum field theory. Comm. Math. Phys. 237, 31-68 (2003).

[7] T. Coquand \& B. Spitters, Integrals and valuations. To appear. http://www.cs.ru.nl/ spitters/integrals.pdf.

[8] M.L. Dalla Chiara \& R. Giuntini, Quantum logics. arXiv:quant-ph/0101028.

[9] R. DiSalle, Understanding space-time: The philosophical development of physics from Newton to Einstein. Cambridge University Press, Cambridge, 2006.

[10] A. Döring \& C.J. Isham, A topos foundation for theories of physics: I. Formal languages for physics. arXiv:quant-ph/0703060. II. Daseinisation and the liberation of quantum theory. arXiv:quant-ph/0703062. III. The representation of physical quantities with arrows.arXiv:quant-ph/0703064. IV. Categories of systems. arXiv:quant-ph/0703066.

[11] J. M. G. Fell, The Dual Spaces of C*-Algebras. Trans. Amer. Math. Soc. 94, 365-403 (1960).

[12] R. Goldblatt, Topoi: The categorial analysis of logic. Second edition. North-Holland, Amsterdam, 1984.

[13] R. Haag \& D. Kastler, An algebraic approach to quantum field theory. J. Math. Phys. 5, 848-861 (1964).

\footnotetext{
${ }^{23}$ According to our previous discussion, one should initially assume that algebras of observables are pre-quasi- $C^{*}$-algebras instead of $C^{*}$-algebras. The completion process is topos-dependent.

${ }^{24}$ Of course, a given noncommutative $C^{*}$-algebra cannot, in general, be mapped into a commutative one in a different topos by a geometric morphism. Similarly, there exists no global diffeomorphism transforming a gravitational field away.

${ }^{25}$ The same is true of the spectral object constructed by Döring and Isham [10].
} 
[14] C. Heunen, N.P. Landsman \& B. Spitters, A topos for algebraic quantum theory. arXiv: 0709.4364 v2.

[15] C. J. Isham \& J. Butterfield, Topos perspective on the Kochen-Specker theorem. I. Quantum states as generalized valuations. Internat. J. Theoret. Phys. 37 , 2669-2733 (1998). J. Butterfield \& C. J. Isham, A topos perspective on the Kochen-Specker theorem. II. Conceptual aspects and classical analogues. Internat. J. Theoret. Phys. 38, 827-859 (1999).

[16] A.J. Kox et al. (eds.), The Collected Papers of Albert Einstein. Volume 6: The Berlin Years: Writings, 1914-1917. Princeton University Press, Princeton, 1996. M. Janssen et al. (eds.), The Collected Papers of Albert Einstein. Volume 7, The Berlin Years: Writings, 1918-1921. Princeton University Press, Princeton, 2002.

[17] P.T. Johnstone, Sketches of an elephant: a topos theory compendium. Vols. 1, 2. Oxford University Press, Oxford, 2002.

[18] J. Kalckar (ed.), Niels Bohr: Collected Works. Vol. 6: Foundations of quantum physics I (19261932). North Holland, Amsterdam, 1985. Vol. 7: Foundations of quantum physics II (1933-1958). North Holland, Amsterdam, 1996.

[19] R. Krömer, Tool and object: a history and philosophy of category theory. Birkhäuser, Basel, 2007.

[20] N.P. Landsman, When champions meet: Rethinking the Bohr-Einstein debate. Stud. Hist. Phil. Mod. Phys. 37, 212-242 (2006). arXiv:quant-ph/0507220.

[21] N.P. Landsman, Between classical and quantum. Handbook of the Philosophy of Science Vol. 2: Philosophy of Physics, eds. J. Butterfield \& J. Earman, pp. 417-554. North-Holland, Elsevier, Amsterdam 2007. arXiv: quant-ph/0506082.

[22] S. Mac Lane \& I. Moerdijk, Sheaves in geometry and logic: A first introduction to topos theory. Springer-Verlag, New York, 1994.

[23] C. McLarty, The uses and abuses of the history of topos theory. British J. Philos. Sci. 41 351-375 (1990).

[24] I. Moerdijk \& G. E. Reyes, Models for smooth infinitesimal analysis. Springer-Verlag, New York, 1991.

[25] J.D. Norton, General covariance and the foundations of general relativity: eight decades of dispute. Reports on Progress in Physics 56, 791-858 (1993).

[26] C. Piron, Foundations of quantum physics. W. A. Benjamin, Reading, 1976.

[27] E. Scheibe, The logical analysis of quantum mechanics. Pergamon Press, Oxford, 1973.

[28] L. Smolin, The trouble with physics. Houghton-Mifflin, Boston, 2006.

[29] S. Vickers, Locales and toposes as spaces. Handbook of Spatial Logic, eds. M. Aiello, I. PrattHartmann, J. van Benthem, Chapter 8. Springer, Heidelberg, 2007.

[30] P. Woit, Not even wrong. Basic Books, New York, 2006. 